\title{
Early rectal cancer accompanied by multiple systemic abscesses: A case report
}

\author{
TAKAYUKI TAJIMA ${ }^{1}$, MASAYA MUKAI $^{2}$, SAYURI HASEGAWA $^{2}$, SOUICHIROU YAMAMOTO $^{2}$, EIJI NOMURA ${ }^{2}$, \\ TAKUMA TAJIRI ${ }^{3}$, SOTARO SADAHIRO $^{1}$, SEIEI YASUDA $^{1}$ and HIROYASU MAKUUCHI ${ }^{1,2}$ \\ ${ }^{1}$ Department of Surgery, Tokai University School of Medicine, Isehara, Kanagawa 259-1193; \\ Departments of ${ }^{2}$ Surgery and ${ }^{3}$ Pathology, Tokai University Hachioji Hospital, Hachioji, Tokyo 192-0032, Japan
}

Received August 18, 2014; Accepted April 21, 2015

DOI: $10.3892 / 01.2015 .3500$

\begin{abstract}
A 70-year-old man undergoing treatment for diabetes presented with a cough and pyrexia that has lasted one week. Laboratory tests demonstrated evidence of inflammation. The patient was hospitalized and antibiotic treatment was initiated, but the condition of the patient did not improve. After 2 weeks, computerized tomography scanning demonstrated the presence of multiple small nodules in the lungs and a liver abscess. The patient also developed neck pain and numbness of the upper extremities and was then transferred to Tokai University Hachioji Hospital (Tokyo, Japan). Percutaneous transhepatic drainage (PTD) of the liver abscess was performed and antibiotic treatment was initiated. Detailed examination revealed there was pyogenic spondylitis of the cervical spine, therefore abscess drainage and an anterior cervical spinal fusion were performed. Culture of each lesion resulted in Klebsiella pneumoniae growth. While continuing antibiotic treatment and rehabilitation, the gastrointestinal tract was investigated and evidence of early rectal cancer was observed. The pulmonary nodules disappeared during treatment, indicating that these were multiple lung abscesses. Four weeks following abscess drainage and anterior cervical spinal fusion, lower anterior resection was performed. The present case report describes a patient who developed multiple abscesses associated with early rectal cancer and discusses the case with reference to the literature.
\end{abstract}

Correspondence to: Dr Takayuki Tajima, Department of Surgery, Tokai University School of Medicine, 143 Shimokasuya, Isehara, Kanagawa 259-1193, Japan

E-mail: takayukitajima1970@yahoo.co.jp

Abbreviations: PTD, percutaneous transhepatic drainage; $\mathrm{Ra}$, above peritoneal reflection

Key words: early colorectal cancer, systemic abscess, pyogenic liver abscess

\section{Introduction}

At present, the global incidence of colorectal cancer is increasing, which is considered to be a result of westernization of the diet and obesity (1-4). The main symptoms are rectal bleeding with or without progressive anemia and weight loss. The recommended treatment for stage I colorectal cancer is surgical resection combined with lymph node dissection (5). The 5-year survival rate of patients following resection of stage I colorectal cancer is $90-95 \%$, with postoperative distant metastasis/recurrence occuring in 5-10\% of patients (5). The incidence of postoperative local recurrence in the pelvic cavity is low (5-7). However, it is not uncommon for colon cancer to cause an abscess in a distant organ (8-16). Regarding the pathogenesis of systemic abscesses associated with colorectal cancer, small abscesses may form around the tumor or bacterial invasion may occur secondary to damage to the intestinal wall, which is followed by hematogenous spread (13). The development of abscesses may be promoted by the impaired immunity of tumor-bearing patients and associated diseases, such as diabetes, may also affect immunity $(12,13)$. In the literature, five cases of colorectal cancer in which liver abscesses were the chief complaint have been reported. Of these five cases, 1 early cancer patient (8) and 3 advanced cancer patients exhibited liver abscesses alone $(9,10,13)$ and 1 advanced cancer patient exhibited multiple systemic abscesses (12). Fever was the main symptom in all cases, with weight loss, malaise and nausea/vomiting also observed $(8-10,12,13)$. In addition, the case of an advanced colorectal cancer patient exhibiting multiple systemic abscesses without any liver abscesses has also been reported (16). However, to the best of our knowledge there have been no reports of multiple systemic abscesses associated with early rectal cancer. The present case study describes the management of a patient with multiple systemic abscesses due to early rectal cancer.

\section{Case report}

The study was approved by the ethics committee of Tokai University Hachioji Hospital (Tokyo, Japan) and all patients provided written, informed consent in accordance with the institutional review board of Tokai University Hachioji Hospital. The patient was a 70-year-old man who presented 


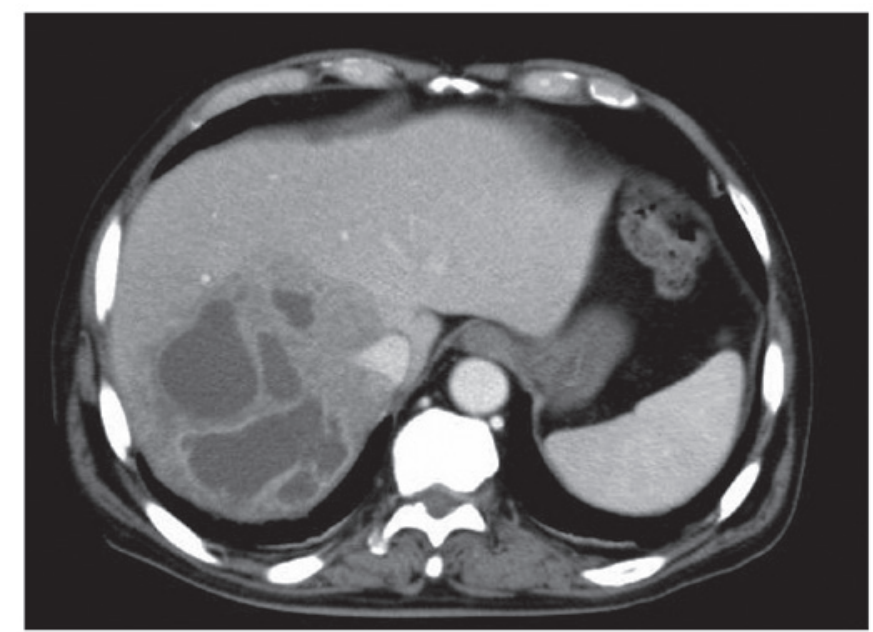

Figure 1. Abdominal contrast-enhanced CT scan. There is a multilocular abscess in the liver associated with low density areas that is surrounded by reactive edema. The abscess wall is enhanced.
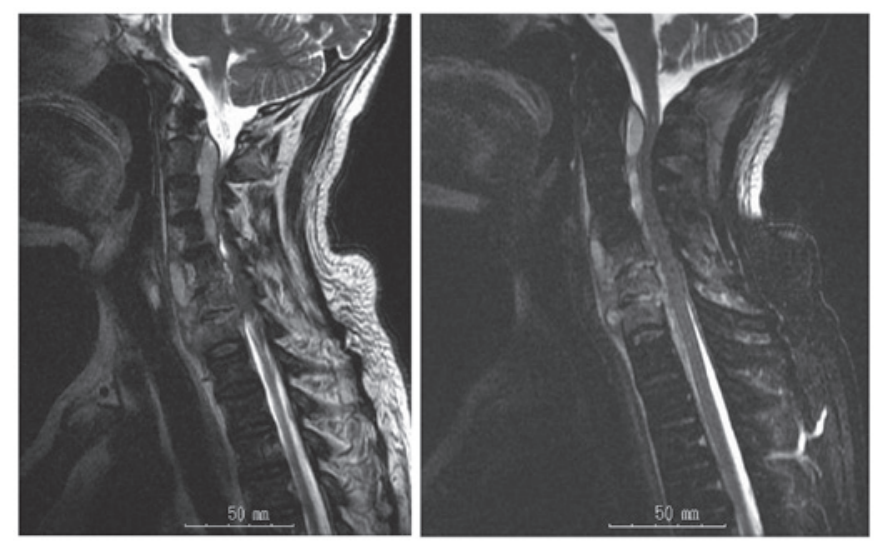

Figure 2. MRI of the cervical region (T2/T2 fat-suppressed image). An epidural abscess extends from $\mathrm{C} 2$ to $\mathrm{C} 7$. Destruction of the vertebral bodies is present at $\mathrm{C} 5$ and $\mathrm{C} 6$.

with cough, fever and numbness of the upper extremities. The patient was diabetic, however that was controlled. In early February 2008, the patient developed a cough and pyrexia. The patient was admitted to Uenohara Municipal Hospital (Yamanasi, Japan) where the patient had been treated for diabetes, but his symptoms did not improve. Computerized tomography (CT) scanning was performed in late February 2008, revealing an abscess in the liver and multiple nodules in the lungs, which were also considered likely to be abscesses. The patient then developed numbness of the upper extremities and neck pain, and was subsequently transferred to Tokai University Hachioji Hospital (Tokyo, Japan). Physical examination indicated that the patient was $161 \mathrm{~cm}$ tall and weighed $64 \mathrm{~kg}$; blood pressure was 136/80 $\mathrm{mmHg}$; pulse rate was 92/min (regular); and body temperature was $38.5^{\circ} \mathrm{C}$. The patient did not have conjunctival pallor or jaundice, and the abdomen was soft and flat. Laboratory blood tests demonstrated a slight increase in the white blood cell count to 9,500/ $\mu 1$ and a high C-reactive protein level of $20.6 \mathrm{mg} / \mathrm{dl}$. In addition, the patient demonstrated elevated levels of aspartate aminotransferase (GOT/AST), alanine aminotransferase (GPT/ALT), alkaline

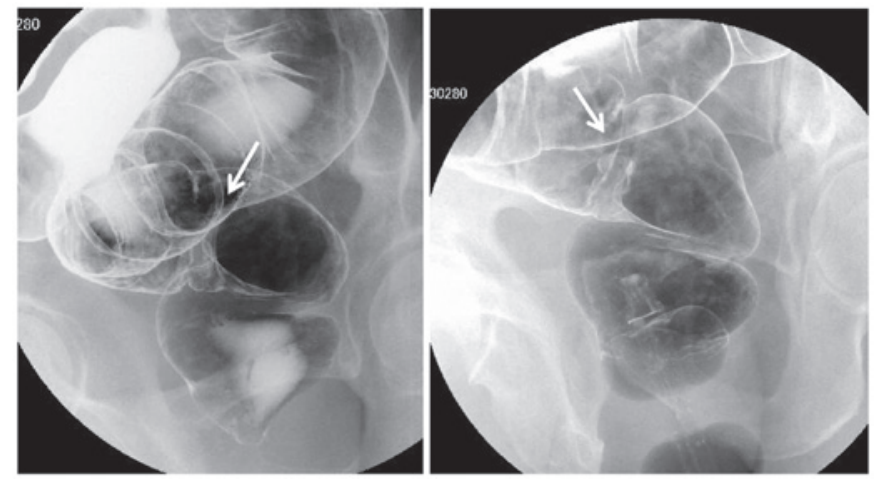

Figure 3. Barium enema. There is a protruding lesion with an irregular border on the wall of the rectum $(\mathrm{Ra})$, indicated by a white arrow.

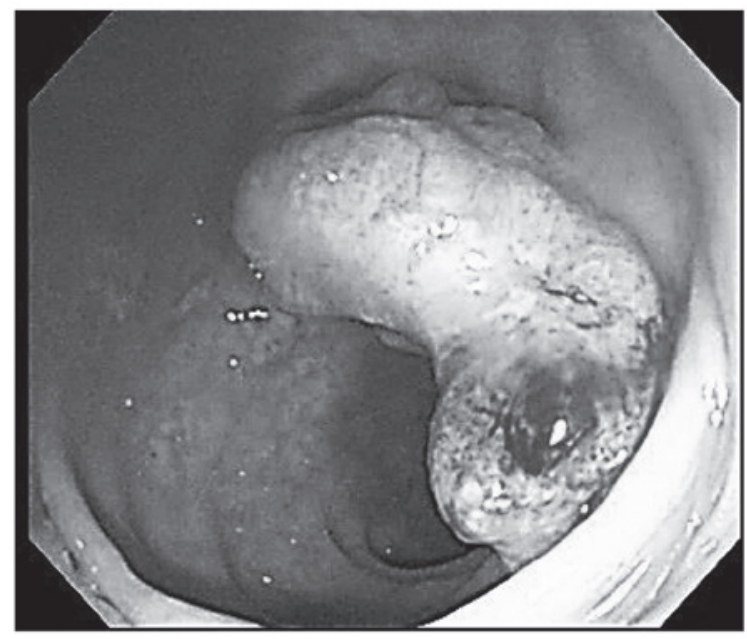

Figure 4. Colonoscopy findings. A protruding lesion with an irregular border is present on the wall of the rectum (Ra). Biopsy indicated that this was a moderately-differentiated adenocarcinoma.

phosphatase (ALP) and $\gamma$-glutamyl transpeptidase $(\gamma$-GTP) to $69,53,650$ and $129 \mathrm{IU} / 1$, respectively. The blood glucose level was high at $210 \mathrm{mg} / \mathrm{dl}$, however the glycated hemoglobin (HbA1c) levels were only slightly elevated to $6.4 \%$, indicating that until recently the patient's diabetes was well controlled. CT scans demonstrated multiple nodular lesions in the lungs, which were considered to be multiple lung abscesses. In addition, there was a multilocular cystic lesion in segments 4,7 and 8 of the liver, which was considered to be a liver abscess (Fig. 1). Abdominal ultrasonography also demonstrated a multilocular cystic lesion in segments 4,7 and 8 of the liver. Magnetic resonance imaging of the cervical region revealed an epidural abscess that extended from $\mathrm{C} 2$ to $\mathrm{C} 7$, originating from a site of vertebral destruction due to spondylitis at C5/6. Accordingly, osteomyelitis of the upper cervical spine was diagnosed (Fig. 2) combined with liver abscess and multiple lung abscesses. Percutaneous transhepatic drainage (PTD) of the liver abscess was immediately performed and $\geq 100 \mathrm{ml}$ of yellowish-white pus was removed. There was no connection between the abscess and the intrahepatic bile ducts. Systemic administration of antibiotics was initiated $(17,18)$. Since the patient also experienced numbness of the upper extremities, drainage of the neck abscess, iliac bone grafting and anterior 

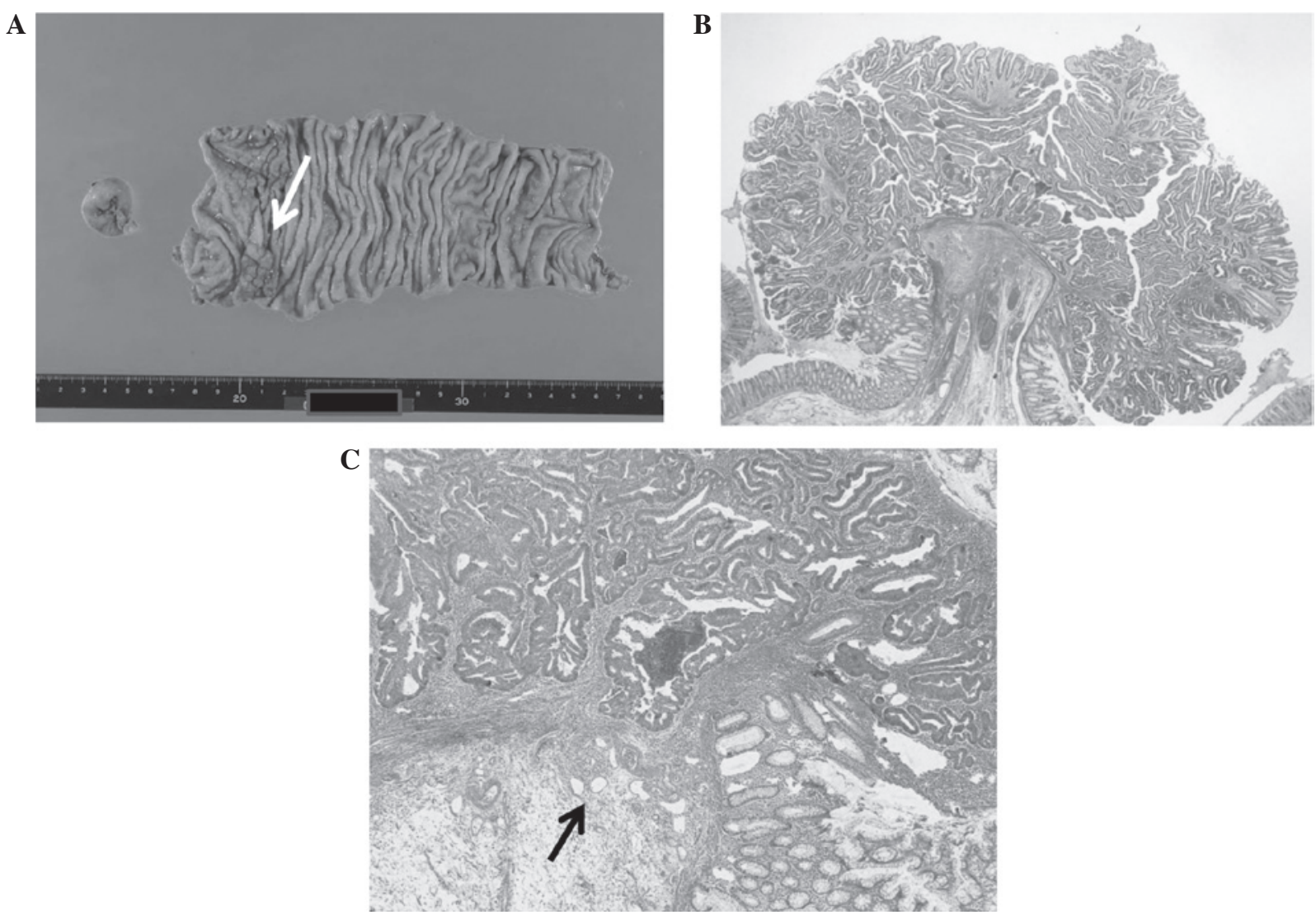

Figure 5. Resected specimen. (A) Resected specimen: There is a protruding lesion with a diameter of 20x10 mm in the rectum (Ra; above peritoneal reflection). White arrow: tumor site. (B) Low-power view: There is a protruding lesion. (C) Histopathological findings: The muscularis mucosa is invaded by moderately differentiated adenocarcinoma in the central area. The actual depth of invasion was 1,500 $\mu \mathrm{m}$. Black arrow: infiltrated region of tumor (hematoxylin and eosin, magnification $\mathrm{x} 200$ ).

cervical spinal fusion were promptly performed. Following these procedures, a thorough examination of the gastrointestinal tract was performed, while continuing antibiotic treatment and rehabilitation. A barium enema revealed a protruding lesion with an irregular border in the rectum ( $\mathrm{Ra}$; above the peritoneal reflection) (Fig. 3). Colonoscopy detected a protruding lesion with an irregular border on a rectal fold. Biopsy of the lesion demonstrated that it was a moderately differentiated adenocarcinoma (Fig. 4). The nodular lesions in the lungs of the patient were no longer detected when CT scanning was performed again once the inflammation had subsided, which indicates that these were multiple lung abscesses. Consequently, the final diagnosis was early rectal cancer that presented with multiple systemic abscesses. Radical resection of the rectal lesion was performed $\sim 1$ month following the initiation of treatment for the abscesses.

Midline laparotomy was performed in the lower abdomen and the peritoneal cavity was investigated but there was no peritoneal dissemination or liver metastasis. The rectal cancer was removed by lower anterior resection. Multiple blocks were taken and routinely processed in paraffin. The sections were stained with hematoxylin and eosin. The pathological diagnosis was made using microscopic examination using a BX51 light microscope with a U-TV0.5XC-3 adaptor, and images were captured using a DP-21 camera (Olympus Corporation, Tokyo, Japan). Examination of the resected specimen indicated an Isp type (subpedunculated) tumor with a diameter of $2 \times 1 \mathrm{~cm}$ in the rectum (Ra; above the peritoneal reflection) (Fig. 5A). The histopathological diagnosis was a moderately differentiated adenocarcinoma, which was Ra, pT1b (early cancer with the deepest level of invasion being 1,500 $\mu \mathrm{m}$ ), ly0, v0, N0 and M0, and the tumor was classified as stage 1 (Fig. 5B and C), as in accordance with the General Rules for Clinical and Pathological Studies on Cancer of the Colon, Rectum and Anus (19). The patient's postoperative course was uneventful and there has been no evidence of metastasis or recurrence in the 7 years following the surgery.

\section{Discussion}

It is uncommon for colorectal cancer to present with liver abscess and this is reported to occur in $\leq 5 \%$ of patients $(20,21)$.The incidence of abscess formation due to colorectal cancer is highest in the liver, followed by the lungs, and cases of endocarditis and meningitis have also been reported (8-16). However, to the best of our knowledge there has only been 1 reported case of early colorectal cancer associated with a liver abscess (1), in addition to 2 cases of multiple abscesses due to colorectal cancer $(12,16)$; therefore, the patient in the present case report is the first to be reported with multiple abscesses caused by early rectal cancer. The majority of the bacteria isolated from abscesses associated with colorectal cancer are gram negative rods such as Klebsiella $(50.0 \%)$ or Fusobacterium $(6.90 \%)$ species $(14,15)$. Klebsiella with the same antibiotic sensitivity profile was isolated 
from the liver and neck abscesses from the patient in the current study. The potential mechanisms of abscess formation include bacteremia as a result of a hematogenous spread from the tumor, direct extension of the intraperitoneal infection along the portal vein or a secondary infection of metastases. Since the patient in the present report was elderly and diabetic, impairment of the immune system may have been associated with the formation of multiple abscesses in addition to the colon cancer $(13,22,23)$. Lonardo et al (13) considered that micro-abscesses may occasionally develop surrounding colon cancer, and lead to bacterial infection in multiple organs by spreading the infection through the portal vein if the defense mechanism of the intestinal tract wall against infection is disrupted. However, the patient in the present study received antibiotic treatment for the liver and cervical abscesses and no micro-abscesses or extensive necrosis were observed in the resected specimen. Nevertheless, the tumor depth had invaded into the submucosal layer $(1,500 \mu \mathrm{m})$, so the possibility of bacterial infection may not be ruled out. Initially, the liver and cervical abscesses were observed in the patient, while the multiple lung nodules disappeared during antibiotic therapy, indicating that these nodules were lung abscesses. It is important to note that multiple abscess formation can be one manifestation of colorectal cancer. Cohen et al (24) and Huang et al (15) reported that it is necessary to investigate the gastrointestinal tract in patients who develop an abscess of unknown etiology.

For management of colorectal cancer associated with an abscess, it is common to initially treat the abscess conservatively with antibiotics and drainage and then perform definitive surgery for the cancer once the abscess has resolved. Since the patient in the present study was experiencing numbness of the upper extremities when transferred to Tokai University Hachioji Hospital, drainage of the cervical spine abscess and iliac bone grafting was performed and immediately followed by anterior cervical spinal fusion. Subsequent to these procedures, systemic antibiotic treatment and rehabilitation was promptly initiated. In considering antibiotic therapy for the patient, the de-escalation method was decided upon as the most effective, and thus, antibiotic agents with a wider antibacterial spectrum were administered initially and then substituted to other agents with a narrower spectrum. Although the optimum timing for radical resection of rectal cancer in these patients has not yet been defined clearly, surgery was performed 4 weeks following transfer of the patient to Tokai University Hachioji Hospital since the patient's general condition had improved. As the patient's postoperative course was uneventful, the timing of the surgery was considered to be appropriate. It is occasionally difficult to differentiate between abscesses and metastasis, but in the present case the multiple lung nodules disappeared following antibiotic treatment, indicating that these lesions were abscesses. However, patients who developed metastases to the lung or liver following surgery have been reported (12), so care should be taken when making a diagnosis. Considering the possibility that an abscess may be caused by secondary infection of a metastasis, it is important to pay close attention to patients who may have metastatic cancer.

In conclusion, the present case emphasizes that it is necessary to perform thorough investigation for possible colorectal cancer (including early cancer) when treating a patient who has multiple abscesses involving organs such as the liver. It is also important to follow the patient carefully following surgery due to the risk of metastasis.

\section{References}

1. Larsson SC and Wolk A: Obesity and colon and rectal cancer risk: A meta-analysis of prospective studies. Am J Clin Nutr 86: 556-565, 2007.

2. Moghaddam AA, Woodward M and Huxley R: Obesity and risk of colorectal cancer: aAmeta-analysis of 31 studies with 70,000 events. Cancer Epidemiol Biomarkers Prev 16: 2533-2547, 2007.

3. No authors listed: Obesity: Preventing and managing the global epidemic. Report of a WHO consultation. World Health Organ Tech Rep Ser 894: 1-253, 2000.

4. Endo H, Higurashi T, Takahashi H and Nakajima A: Obesity, life style-related diseases and colorectal cancer. Gastro Endosc 55: 3735-3744, 2013 (In Japanese).

5. Watanabe T, Itabashi M, Shimada Y, et al; Japanese Society for Cancer of the Colon and Rectum: Japanese Society for Cancer of the Colon and Rectum (JSCCR) Guidelines 2014 for treatment of colorectal cancer. Int J Clin Oncol 20: 207-239, 2015.

6. Journal of Health and Welfare Statistics, Health and Welfare Statistics Association, Tokyo, 61: 63-67, 2014/2015.

7. Multi-Institutional Registry of Large Bowel Cancer in Japan. Cases treated in 1994. Japanese Society for Cancer of the Colon and Rectum, Tokyo, 23, 2002.

8. Tanizaki H, Kawano N, Watanabe H, Tsutsumi O, Hatate K and Sugai T: A case of a solitary liver abscess complicated by early sigmoid colon cancer. J Jpn Surg Assoc 63: 449-453, 2002. (In Japanese).

9. Ritchie JD: Portal pyaemia secondary to carcinoma of the rectum. Aust NZJ Surg 45: 284-285, 1975.

10. Panwalker AP: Unusual infections associated with colorectal cancer. Rev Infect Dis 10: 347-364, 1988.

11. Legier JF: Streptococcus salivarius meningitis and colonic carcinoma. South Med J 84: 1058-1059, 1991.

12. Hiratsuka $H$, Yasui $N$ and Maeda $K$ : A case of cancer of the rectum accompanied with multiple systemic abscesses. JJSC 65: 453-457, 2012. (In Japanese).

13. Lonardo A, Grisendi A, Pulvirenti M, et al: Right colon adenocarcinoma presenting as Bacteroides fragilis liver abscesses. J Clin Gastroenterol 14: 335-338, 1992.

14. Qu K, Liu C, Wang ZX, Tian F, et al: Pyogenic liver abscesses associated with nonmetastatic colorectal cancers: an increasing problem in Eastern Asia. World J Gastroenterol 18: 2948-2955, 2012.

15. Huang WK, Chang JW, See LC, et al: Higher rate of colorectal cancer among patients with pyogenic liver abscess with Klebsiella pneumoniae than those without: an 11-year follow-up study. Colorectal Dis 14: e794-e801, 2012.

16. Isohata N, Watanabe O, Domoto $\mathrm{K}$, et al: A case of rectal cancer developed bacterial meningitis. JJCS 32: 783-786, 2007. (In Japanese).

17. Andersson R, Forsberg L, Hederstrom E, Hochbergs P and Bengmark S: Percutaneous management of pyogenic hepatic abscesses. HPB Surg 2: 185-188, 1990.

18. Nosher JL, Giudici M, Needell GS and Brolin RE: Elective one-stage abdominal operations after percutaneous catheter drainage of pyogenic liver abscess. Am Surg 59: 658-663, 1993.

19. Japanese Society for Cancer of the Colon and Rectum (JSCCR): General Rules for Clinical and Pathological Studies on Cancer of the Colon, Rectum and Anus. 7th edition, revised version. Kanehara Shuppan, Tokyo, Japan, 2009.

20. McDonald AP and Howard RJ: Pyogenic liver abscess. World J Surg 4: 369-380, 1980

21. McDonald MI, Corey GR, Gallis HA and Durack DT: Single and multiple pyogenic liver abscesses. Natural history, diagnosis and treatment, with emphasis on percutaneous drainage. Medicine (Baltimore) 63: 291-302, 1984.

22. Teitz S, Guidetti-Sharon A, Manor H and Halevy A: Pyogenic liver abscess: warning indicator of silent colonic cancer. Report of a case and review of the literature. Dis Colon Rectum 38: 1220-1223, 1995.

23. Nosher JL, Giudici M, Needell GS and Brolin RE: Elective one-stage abdominal operations after percutaneous catheter drainage of pyogenic liver abscess. Am Surg 59: 658-663, 1993.

24. Cohen JL, Martin M, Rossi RL, and Schoetz DJ: Liver abscess. The need for complete gastrointestinal evaluation. Arch Surg 124: 561-564, 1989. 